\title{
A Study on Flexible Vibratory Feeding System Based on HALCON Machine Vision Software
}

\author{
Liang $\operatorname{Han}^{\mathrm{a}}$, Jie Deng ${ }^{\mathrm{b}}$ \\ School of Mechanical Engineering, Southeast University, Jiangning District, \\ Nanjing, 211189, China \\ amelhan@seu.edu.cn, ${ }^{b}$ dengjie5121226@126.com
}

Keywords: Vibratory Feeding; Machine Vision; HALCON; Flexibility; Orientation

\begin{abstract}
Vibratory feeder, thanks to its excellent material storage, workpiece orientation and continuous feeding characteristics, is widely used in industrial automation. Since its versatility is not strong, orienting mechanism needs to be designed for different workpieces, which often cannot meet today's rapidly changing market. In light of this, this paper introduces a flexible vibratory feeding system based on machine vision. On the basis of traditional vibratory feeder, workpiece orientation can be achieved with machine vision and appropriate executive mechanism. In this paper, HALCON and industrial cameras are used to build a machine vision detection platform, HALCON program is written for detecting the orientation of workpiece, and the experimental results are promising.
\end{abstract}

\section{Introduction}

Vibratory feeder, as an important part of vibratory feeding system, is widely used in electronic components, tobacco, automotive parts and other industries automated assembling line [1]. The vibratory feeder is mainly used for material storage and orienting feeding, among which orienting feeding is very important. The main orienting mechanism of traditional vibratory feeder includes blade, track, step, hole, groove, etc., whose design usually utilizes the gravity of workpieces [2] [3]. The corresponding orienting mechanism needs to be designed for different workpieces. Especially for complex workpiece, the design of orienting mechanism often requires repeated adjustment, which relies on the experience of producers [4].

In view of the problems of traditional vibratory feeder, now, we solve them mainly by combining machine vision with vibratory feeder. Just by writing corresponding program, it can not only realize the orientation of different workpieces, but also detect surface of workpiece, which is unable to be achieved by traditional orientation mechanism [5]. In the eighties of last century, American Artificial Intelligence Center, John W Hill developed a programmable vibratory feeder based on machine vision [6]. At the beginning of this century, Nanyang Technological University, Singapore, Sim S K applied neural network to vibratory feeder workpiece orientation through improving algorithm, which achieved good results [7]. While in China, the study on applying machine vision to vibratory feeder workpiece orientation is still in initial stage. At the same time, the machine vision algorithm is mostly written by MATLAB or directly uses OpenCV - an open source computer vision and machine learning software library, which often cannot be compared with the mature commercial algorithm library on the flexibility of development, efficiency of algorithm as well as development cycle.

In this paper, commercial machine vision algorithm library - HALCON, vibratory feeder and corresponding execution mechanism are selected to build a flexible vibratory feeding system. 


\section{Main composition of the system}

The flexible vibratory feeding system based on HALCON is shown in Figure 1. It mainly contains vibratory feeding system, machine vision system and executive mechanism, among which vibratory feeding system is the foundation, machine vision detection technology is the key, executive mechanism is a necessary condition to realize orientation function. Figure 2 shows the experimental device.

\subsection{Design of Vibratory feeding system}

The vibratory feeding system mainly includes vibratory feeder and the frame. The vibratory feeder sends workpiece to the testing area, the frame supports vibratory feeder, camera, light, etc.

In order to meet the needs of the machine vision platform, this paper designs a special vibratory feeder. The track is coated with Teflon to improve the wear resistance of the track and to highlight the contrast between workpiece and background. The matte finishing is taken to reduce the reflection. At the same time, damping treatment is done between frame and the vibratory feeder to reduce influence of frame vibration on the camera image acquisition.

\subsection{Design of Machine vision system}

Machine vision system is the key part of the whole system, the identification and detection of workpiece entirely depend on the machine vision algorithm. Therefore, calculation accuracy, processing efficiency, programming flexibility, development cycle, and hardware platform of machine vision system are very important. Machine vision system mainly includes software platform and hardware platform [8] [9] [10]. The hardware mainly contains light source, lens, camera, IPC etc. The software contains machine vision algorithm library, software development platform and operating system. While designing, the number and execution efficiency of the algorithm, efficiency of the software development platform and development cycle are taken into the main consideration [11] [12].

HALCON is one of the most successful commercial machine vision algorithms library. HALCON has a powerful integrated development environment and includes over 1600 operator functions, such as blob analysis, morphological analysis, template matching, etc. HALCON has efficient execution speed and supports multi-core CPU and GPU acceleration. HALCON has high portability across platforms and supports Windows, Linux, Solaris operating system, and can be easily called in C, $\mathrm{C}++, \mathrm{C}$ \#, Visual Basic.NET, Delphi and other programming languages [13].

In this paper, the camera model is AVT-GC2450, the light source is a white ring light, IPC's CPU is Intel Core i7, software platform uses HALCON algorithm library and Visual Studio 2010 C ++ MFC.

\subsection{Design of executive mechanism}

The executive mechanism is an important part of system. It mainly consists of single chip microcomputer, electromagnetic valve and jet nozzle. The microcontroller communicates with the computer through the serial port and controls the electromagnetic valve. 


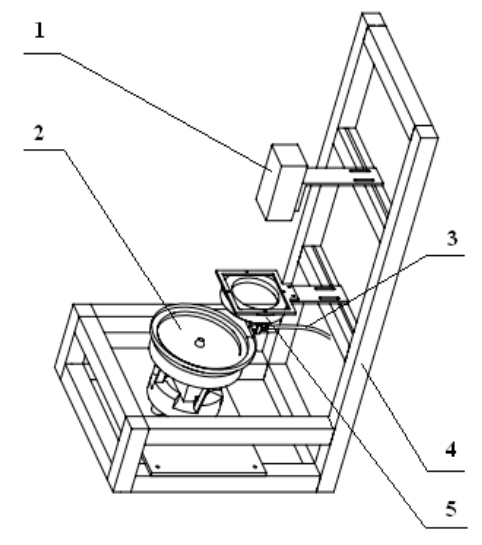

Figure 1. The Overall Design

1 CCD camera 2 Vibratory feeder

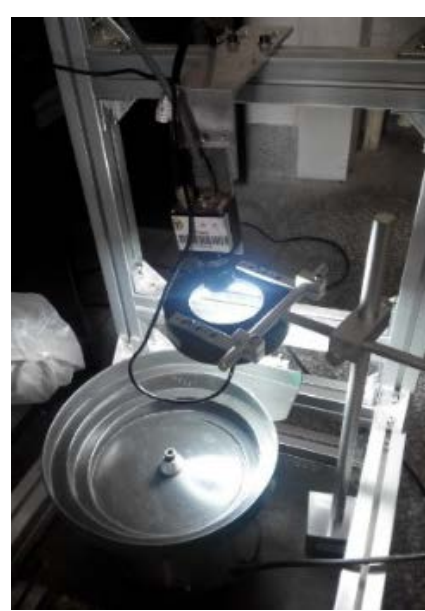

Figure 2. Experimental device

3 Executive mechanism 4 Frame 5 Light source

\section{Workpiece recognition experiment}

According to the above choice, a nut is selected as workpiece to build the experiment platform. Considering the workpiece has regular shape and can be easily segmented from background, we can judge workpiece orientation by calculating smallest enclosing rectangle of workpiece.

The detailed procedure includes: Image acquisition - Threshold segmentation - Morphological opening operation - Connected region computing - Region extraction - Smallest enclosing rectangle calculating. The implementation effect is shown in Table 1.

Considering the workpiece is regular rectangle, and has big differences between its length and width, we can judge workpiece orientation according to the values of $\mathrm{X}, \mathrm{Y}$ and Phi. Table 2 lists the results of workpiece on several different orientations. The results show that the values of $\mathrm{X}, \mathrm{Y}$ and Phi have big difference when workpiece is on different orientation. So this is an easy way to judge workpiece orientation.

Table 1. Image Segmentation Processing Steps

\begin{tabular}{|c|c|c|}
\hline Original Image & Divided & Smallest enclosing rectangle \\
\hline & & \\
\hline
\end{tabular}

Table 2. Results of the Workpiece at Several Different Orientations

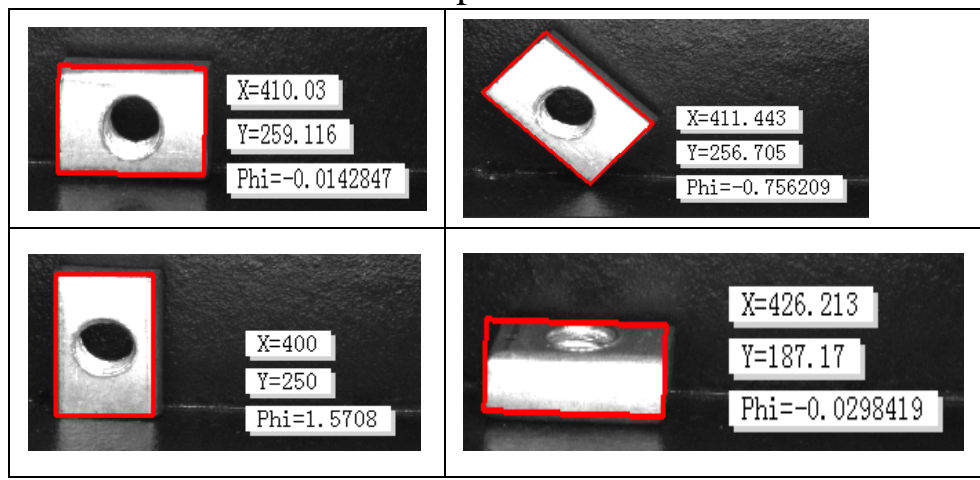

Note: Phi is the angle of rectangle and horizontal axis, $\mathrm{X}$ is the length of long edge of the rectangle, $\mathrm{Y}$ is the length of short edge of the rectangle, the unit of $\mathrm{X}$ and $\mathrm{Y}$ is pixel, the unit of Phi is rad. 


\section{Summary}

In this paper, on basis of the traditional vibratory feeder, we build a vibratory feeding system by selecting appropriate camera, light source, software and the corresponding executive mechanism. Through writing appropriate program, workpiece orientation can be easily judged according to the results. Also relying on HALCON's high efficiency, rapid and integrated development platform, it can quickly prepare workpiece detection program. On the same hardware platform, just by writing corresponding program, it can meet the needs of different workpiece orientation, which makes the vibratory feeding system programmable and intelligent. Through continuous improvement of the machine vision algorithm, we can also do research on workpiece surface inspection, foreign object detection and workpiece motion state detection.

\section{Acknowledgement}

This work is supported by the NSFC, project No.: 51275087.

\section{References}

[1] Boothroyd G, Polio C, Murch L E. Automatic Assembly. New York: Marcel Dekker, 1982

[2] L Han, C B Li, G P Hu. A Study on the Vision-Based Flexible Vibratory Feeding System. Advanced Materials Research, 2011, 279: 434-439

[3] H G de Cock. Vibratory Feeder. Philios Technical Review, 1962, 24(3):84-95

[4] L Han, Xu Wei-liang. Research Status and Prospect of Vibratory Feeder [J]. Special Equipment of Electronic Industry, 1999 (1): 6-13

[5] Cai Chen-yun, Li Xia. State Correction Equipment of Work-piece Based on Machine Vision. Journal of Shaanxi University of Science \& Technology, Feb 2011, 29: 20-24

[6] John W Hill. Programmable bowl feeder design based on computer vision. Assembly Module with Vision Controlled Placement Device. Robot Vision, IFS Ltd. UK, 1983:246-251

[7] Sim S K, Chua Patrick S K, Tay M L, etal. The performance of ARTMAP in pattern recognition for a flexible vibratory bowl feeder system. International Conference on Control and Automation, 2003: 223-227

[8] Han Jiu-qiang, Hu Huai-zhong, Zhang Xin-man, etc. Machine Vision Technology and Its Applications [M]. Beijing: Higher Education Press, 2009

[9] Ramesh Jain, Rangachar Kasturi, Brian G. Schunck. MACHINE VISION. Published by McGraw-Hill, Inc., 1995

[10] Carsten Steger, Markus Ulrich, Christian Wiedemann. Machine Vision Algorithms and Applications 2007

[11] Nello Zuech, Richard K. Miller. Machine vision. Lilburn, GA: Fairmont Press, c1987.

[12] Jorge L.C. Sanz. Advances in machine vision. New York: Springer-Verlag, c1989.

[13] HDevelop User's Guide [EB/OE]. http://www.halcon.com/ 